10,04

\title{
Фононы и кроссовер Иоффе-Регеля в случайных решетках произвольной размерности
}

\author{
() Д.А. Конюх \\ Физико-технический институт им. А.Ф. Иофрфе РАН, \\ Санкт-Петербург, Россия \\ E-mail: conyuh.dmitrij@yandex.ru \\ Поступила в Редакцию 8 июля 2021 г. \\ В окончательной редакции 13 июля 2021 г. \\ Принята к публикации 16 июля 2021 г.
}

\begin{abstract}
В рамках подхода теории случайных матриц показано, что релаксация проекции исходной плоской волны с волновым вектором q описывается уравнением движения с запаздывающей частью в виде комплексного динамического модуля Юнга $E(\omega)$. В гармонической скалярной модели смещений в отсутствие диссипации энергии кроссовер Иоффе-Регеля возникает универсальным образом в аморфных системах с размерностью $d \geq 3$. Колебания выше кроссовера Иоффе-Регеля имеют диффузионную природу и могут быть описаны уравнением диффузии с затуханием $\Gamma(\mathbf{q}) \propto q^{2}$.
\end{abstract}

Ключевые слова: аморфные тела, диффузия колебаний, случайные матрицы.

DOI: 10.21883/FTT.2021.12.51658.06s

\section{1. Введение}

Аморфные диэлектрики обладают рядом колебательных свойств, которые принципиальным образом отличают такие неупорядоченные системы от кристаллических. Процессы релаксации колебаний, влияющие на эти свойства, играют важную роль при исследовании аморфных систем [1]. Низкочастотная область колебаний аморфных сред выражена фононами с большой длиной свободного пробега. Однако в широком диапазоне частот ниже порога локализации колебания распространяются посредством диффузионной передачи энергии от атома к атому $[2,3]$. Эти колебания отвечают за вторичный рост теплопроводности аморфных диэлектриков при температурах от 20 до $1000 \mathrm{~K}$ [1]. Кроссовер между низкочастотными фононами и более высокочастотными диффузными колебаниями известен как кроссовер Иоффе-Регеля, при котором длина свободного пробега фонона становится сравнимой с его длиной волны [3].

Практически во всех стеклах вне зависимости от их химического состава наблюдаются универсальные колебательные особенности вблизи кроссовера ИоффеРегеля [4,5]. Это бозонный пик, характеризующий избыточную плотность колебательных состояний $g(\omega)$ по сравнению с законом Дебая, согласно которому низкочастотная плотность состояний пропорциональна $\omega^{d-1}$, где $d$ - размерность пространства [6]. Пик, наблюдаемый в приведенной плотности состояний $g(\omega) / \omega^{d-1}$ во многих экспериментах [7-10], и получил название бозонного пика. Кроме этого, данные по неупругому рентгеновскому рассеянию в стеклах показывают диффузионную особенность затухания $\Gamma(\mathbf{q}) \propto q^{2}$ выше кроссовера Иоффе-Регеля по сравнению с рэлеевской зависимостью $\Gamma(\mathbf{q}) \propto q^{d+1}$ ниже кроссовера [11-13]. Также отмечается линейная корреляция частоты кроссовера Иоффе-Регеля $\omega_{\text {IR }}$ с упругими модулями аморфной среды $[14,15]$. В ряде экспериментальных работ, посвященных исследованию колебаний в двумерных системах, наблюдаются все вышеперечисленные особенности [16-19]. Поэтому описание кроссовера Иоффе-Регеля дает ключ к пониманию процессов колебаний в неупорядоченных средах.

Существуют разные теоретические подходы, используемые для описания данных феноменов аморфных сред [20-25]. Одним из таких подходов является применение теории случайных матриц [26-29]. Можно предполагать, что в аморфных телах, вследствие локального беспорядка, элементы динамической матрицы системы до некоторой степени случайны [30]. В то же время, ближний порядок в стеклах напоминает ближний порядок в соответствующих кристаллах, однако дальний порядок отсутствует. Это позволяет рассматривать только ближнее взаимодействие атомов системы, расположенных при этом в узлах решетки соответствующего кристалла [30]. Такое рассмотрение сильноразреженной случайной динамической матрицы позволяет изучить приведенные особенности колебаний в некоторых двумерных и трехмерных неупорядоченных системах, как с численной, так и с аналитической точек зрения [29,31-33].

Целью настоящей работы является аналитическое описание диффузии колебаний и кроссовера ИоффеРегеля с помощью теории случайных матриц. При этом найденные выражения и выводы не привязаны к какойто конкретной неупорядоченной системе (аморфному телу), а носят обобщающий характер для аморфных сред с произвольным локальным расположением атомов и размерностью. В частности, изучение колебательных свойств неупорядоченных систем с разной размерно- 
стью представляет интерес при исследовании квазилокальных колебаний. Вклад квазилокальных колебаний в плотность состояний $g_{\mathrm{qlv}} \propto \omega^{\beta}, \beta \approx 4$ для большинства стекол $[34,35]$ не зависит от размерности системы. Изучение систем с размерностью $d>3$ позволяет отличить моды этих колебаний от рэлеевского рассеяния фононов на беспорядке, которое дает вклад $g \propto \omega^{d+1}$.

\section{2. Представление динамической матрицы $\hat{M}$ в виде коррелированного ансамбля Вишарта}

Динамическая матрица $\hat{M}$ строится с учетом основных симметрийных свойств механической неупорядоченной системы. Рассматриваемая модель описывает гармонические колебания системы, состоящей из большого числа частиц $N$ вблизи их положения равновесия без диссипации энергии. Для упрощения полагается, что атомы обладают единичными массами, и смещения атомов являются скалярными величинами [29]. В этом случае число степеней свободы системы совпадает с числом атомов $N$. Динамическая матрица $\hat{M}$ связана с полной энергией взаимодействия частиц такой системы $U$ следующим образом [36]:

$$
M_{i j}=\frac{\partial^{2} U}{\partial u_{i} \partial u_{j}},
$$

где индексы $i$ и $j$ нумеруют атомы (степени свободы), $u_{i}$ - скалярное смещение $i$-го атома из положения равновесия.

Нахождение системы вблизи устойчивого положения равновесия означает положительную определенность динамической матрицы $\hat{M}[30]$, поэтому ее можно представить в следующем виде:

$$
\hat{M}=\hat{A} \hat{A}^{T}
$$

где $\hat{A}$ - матрица размером $N$ на $K$. Каждая строка матрицы $\hat{A}$ - соответствует степени свободы системы, а каждый ее столбец соответствует связи некоторого числа степеней свободы между собой [31]. Вследствие локальной флуктуации связей системы можно считать, что элементы матрицы $\hat{A}-$ случайные числа, имеющие гауссово распределение [30]. Тогда (2) соответствует представлению динамической матрицы $\hat{M}$ в виде ансамбля Вишарта случайных матриц. Такая модель описывает многие универсальные колебательные свойства неупорядоченных систем [29,33]. При этом механическая устойчивость системы не накладывает ограничений на вид матрицы $\hat{A}$.

Соотношение числа связей $K$ и числа степеней свободы $N$ играет важную роль при исследовании колебаний механических систем [37,38]. В данной модели рассматривается параметр $\varkappa=K / N-1$, показывающий относительное увеличение числа связей над числом степеней свободы системы. При $\varkappa \leq 0$ система не обладает макроскопической жесткостью в соответствии с правилом Максвелла [39]. При $\varkappa>0$ система обладает конечной макроскопической жесткостью, вследствие чего низкочастотные колебания системы существуют в виде плоских волн и подчиняются закону Дебая [29]. Случай $\varkappa=0$ соответствует предельно мягкой системе, в которой все колебания распространяются посредством некоторой диффузионной передачи энергии от атома к атому [29]. Будем варьировать параметр системы $\varkappa$ в широких пределах $0 \leq \varkappa<\infty$.

Колебания механической системы не зависят от ее сдвига как целой, в результате чего симметричная динамическая матрица должна подчиняться правилу сумм: $\sum_{i} M_{i j}=\sum_{j} M_{i j}=0$ [36]. Это приводит к правилу $\sum_{i} A_{i j}=0$, в результате которого элементы матрицы $\hat{A}$ - являются коррелированными. Статистические свойства некоррелированного ансамбля Вишарта хорошо изучены в рамках теории случайных матриц [40,41]. Однако правило сумм приводит к более сложной задаче о нахождении спектральных свойств коррелированного ансамбля Вишарта. Тем не менее, учет правила сумм является важным, поскольку обеспечивает наличие низкочастотных колебаний в виде плоских волн, подчиняющихся закону Дебая [29].

Для учета этих корреляций будем считать, что все связи статистически одинаковы и независимы, то есть отдельные столбцы матрицы $\hat{A}$ не коррелируют друг с другом. Тогда парные корреляции между матричными элементами $A_{i j}$ имеют вид

$$
\left\langle A_{i k} A_{j l}\right\rangle=\frac{1}{N} C_{i j} \delta_{k l}
$$

где $\hat{C}$ - корреляционная матрица, скобки $\langle\cdot\rangle$ означают усреднение по различным реализациям динамической матрицы $\hat{M}$. Усредненная по ансамблю динамическая матрица связана с матрицей корреляций

$$
\left\langle M_{i j}\right\rangle=\left\langle\Sigma_{k} A_{i k} A_{j k}\right\rangle=K\left\langle A_{i k} A_{j k}\right\rangle=\frac{K}{N} C_{i j} .
$$

Если число связей $K$ существенно превосходит число степеней свободы $N$, то динамическая матрица $\hat{M}$ слабо отличается от усредненной матрицы $\langle\hat{M}\rangle=(\varkappa+1) \hat{C}$. Поэтому интересным представляется случай малой разницы $K$ и $N$, то есть случай $\varkappa \ll 1$.

Статистические свойства случайной матрицы $\hat{M}$ связаны со статистическими свойствами матрицы корреляций $\hat{C}$ [42]. Можно ожидать, что элементы усредненной по различным реализациям динамической матрицы имеют одинаковые значения, вследствие чего матрица корреляций описывает регулярную систему [42]. Тогда волновой вектор системы $|\mathbf{q}\rangle$ является собственным вектором матрицы $\hat{C}$, а ее собственные значения соответствуют квадратам частот колебаний регулярной системы $\omega_{\text {cor }}^{2}(\mathbf{q})$. В работах $[32,33]$ были рассмотрены регулярные системы, соответствующие двумерной и трехмерной 
кубическим решеткам, для которых дисперсионная зависимость $\omega_{\text {cor }}(\mathbf{q})$ является известной. В данной работе считается, что в общем случае при малых волновых векторах $\omega_{\text {cor }}(\mathbf{q}) \propto q$ вне зависимости от конкретного вида матрицы корреляций и размерности системы.

\section{3. Динамический модуль Юнга}

Покажем, что движение профиля смещений атомов $u_{\mathbf{q}}(t)$ может быть описано с помощью уравнения движения с запаздывающей частью. Построенная динамическая матрица $\hat{M}(2)$ описывает малые гармонические колебания в неупорядоченной системе в виде уравнения движения

$$
|\ddot{u}(t)\rangle=-\hat{M}|u(t)\rangle,
$$

где $N$-мерный вектор $|u(t)\rangle$ описывает отклонение атомов от их положения равновесия в момент времени $t$. Уравнение движения (5) может быть решено при задании начальных условий $|u(0)\rangle=0,|\dot{u}(0)\rangle=v_{0}|\mathbf{q}\rangle$.

$\mathrm{B}$ то же время, за счет перераспределения энергии между внутренними степенями свободы системы, проекция $\langle u(t) \mid \mathbf{q}\rangle$ плоской волны с волновым вектором q может испытывать релаксацию. Для изучения этого процесса рассмотрим усредненную проекцию плоской волны $u_{\mathbf{q}}(t)=\overline{\langle u(t) \mid \mathbf{q}\rangle}$, которая связана с функцией Грина $G_{\mathbf{q}}(z)$ динамической матрицы в виде Фурье-преобразования

$$
\begin{gathered}
u_{\mathbf{q}}(t)=-\frac{v_{0}}{2 \pi} \int_{-\infty}^{\infty} G_{\mathbf{q}}\left((\omega-i 0)^{2}\right) e^{i \omega t} d \omega, \\
G_{\mathbf{q}}(z)=\overline{\sum_{n} \frac{|\langle n \mid q\rangle|^{2}}{z-\omega_{n}^{2}}} .
\end{gathered}
$$

Частота $\omega_{n}$ соответствует собственному состоянию $|n\rangle, z$ - комплексная переменная.

В работах $[33,43]$ показано, что в термодинамическом пределе $N \rightarrow \infty$ функция Грина $G_{\mathbf{q}}(z)$ имеет следующий вид:

$$
G_{\mathbf{q}}(z)=\frac{1}{z-\omega_{\mathrm{cor}}^{2}(\mathbf{q}) E(z)},
$$

где комплексная функция $E(z)=z / Z(z)$ в скалярной модели смещений играет роль динамического комплексного модуля Юнга, вещественная часть которого $\operatorname{Re} E(z)$ описывает упругость, а мнимая часть $\operatorname{Im} E(z)-$ вязкость. При этом комплексные параметры $z$ и $Z$ связаны конформным преобразованием $Z(z)$ следующего вида [44]:

$$
\varkappa Z+\frac{Z^{2}}{N} \operatorname{Tr} \hat{R}_{\text {cor }}(Z)=z,
$$

где резольвента $\hat{R}_{\text {cor }}(Z)$ соответствует резольвенте матрицы корреляций

$$
\hat{R}_{\text {cor }}(Z)=\sum_{q} \frac{|\mathbf{q}\rangle\langle\mathbf{q}|}{Z-\omega_{\text {cor }}^{2}(\mathbf{q})} .
$$

Такой вид функции Грина (8) соответствует уравнению движения усредненной проекции плоской волны $u_{\mathbf{q}}(t)$ с запаздывающей частью

$$
\ddot{u}_{\mathbf{q}}(t)+\omega_{\mathrm{cor}}^{2}(\mathbf{q}) \int_{-\infty}^{t} \mathscr{K}(t-\tau) u_{\mathbf{q}}(\tau) d \tau=0,
$$

в котором запаздывающее ядро является Фурьепреобразованием динамического модуля Юнга

$$
\mathscr{K}(t-\tau)=\frac{1}{2 \pi} \int_{-\infty}^{\infty} E\left((\omega-i 0)^{2}\right) e^{i \omega(t-\tau)} d \omega
$$

Комплексный динамический модуль Юнга $E(z)$ может быть найден из конформного преобразования (9) при нахождении зависимости $Z(z)$ в явном виде. Эта зависимость, вообще говоря, является особенной для конкретной регулярной системы, описываемой матрицей корреляций $\hat{C}$, с соответствующей дисперсией $\omega_{\text {cor }}^{2}(\mathbf{q})$. В следующем разделе, исходя из достаточно общих предположений о свойствах большинства таких систем, выводится приближенный общий вид $E(z)$.

\section{4. Низкочастотное приближение}

С помощью ряда приближений универсальным образом удается получить запаздывающую часть и уравнение движения профиля смещений в явном аналитическом виде. Нормированный след резольвенты (10) матрицы корреляций может зависеть от комплексного параметpa $Z$ сложным образом. Эта зависимость определяется конкретным видом вспомогательной регулярной системы, описываемой матрицей корреляций. Однако будем считать, что низкочастотная область колебаний такой системы вне зависимости от ее конкретного вида и размерности обусловлена фононами с линейным законом дисперсии $\omega_{\text {соr }}(\mathbf{q})=\Omega q$ при $q \rightarrow 0$. Постоянная $\Omega$ определяет характерную частоту колебаний системы.

В двумерных системах $d=2$ с низкочастотным линейным законом дисперсии имеется логарифмическая расходимость $N^{-1} \operatorname{Tr} \hat{R}_{\text {cor }}(Z) \simeq \ln (-Z)+O(Z)$ при малых $Z \rightarrow 0$. Такая особенность двумерных систем связана с их постоянной плотностью распределения квадратов частот вблизи нуля. Однако в системах с размерностью $d \geq 3$ разложение резольвенты имеет общий вид $N^{-1} \operatorname{Tr} \hat{R}_{\text {cor }}(Z)=-a^{2}+f(Z)$, где константа $a$ не зависит от $Z$. Например, для простой трехмерной кубической решетки константа $a \approx 0.505462$ (постоянная Ватсона) [45], и функция $f(Z) \simeq \sqrt{-Z}+O(Z)$ имеет корневую особенность при малых $Z$ [46]. Поэтому в первом приближении при $Z \rightarrow 0$ ограничимся только константой: $N^{-1} \operatorname{Tr} \hat{R}_{\text {cor }}(Z) \simeq-a^{2}$. Такое приближение справедливо при $\varkappa \ll 1$ и в общем случае подойдет для качественного анализа систем с размерностью $d \geq 3$, в которых на низких частотах распространяются фононы с линейным 
законом дисперсии. Однако для выявления особенностей конкретных систем необходимо искать вид $f(Z)$.

C учетом описанных низкочастотных приближений, конформное преобразование (9) переписывается в виде

$$
\varkappa-a^{2} \frac{z^{2}}{E(z)}=E(z) .
$$

Ввиду зависимости (6), для каждого комплексного $z=(\omega-i 0)^{2}$ из уравнения (13) можно найти явный вид динамического модуля Юнга $E\left((\omega-i 0)^{2}\right)$. Для простоты опустим $-i 0$ в последующих уравнениях и выберем комплексное решение, которое соответствует положительной плотности колебательных состояний $g(\omega)>0$ :

$$
E(\omega)=\frac{\kappa}{2}+\sqrt{\frac{\kappa^{2}}{4}-a^{2} \omega^{2}}
$$

Статический модуль Юнга $E(0)=\varkappa$ определяет макроскопическую жесткость среды. Тогда уравнение движения (11) принимает следующий вид:

$$
\begin{gathered}
\ddot{u}_{\mathbf{q}}(t)+\frac{\Omega^{2} \kappa}{2} \Delta u_{\mathbf{q}}(t)+\Omega^{2} a \Delta \dot{u}_{\mathbf{q}}(t) \\
+\int_{-\infty}^{t} F(\tau) \Delta u_{\mathbf{q}}(t-\tau) d \tau=0, \\
F(\tau)=\frac{\kappa \Omega^{2}}{2 \tau} J_{1}\left(\frac{\kappa \tau}{2 a}\right),
\end{gathered}
$$

где $\Delta$ - дискретный оператор Лапласа, действующий на проекцию исходной плоской волны с волновым вектором q. Такая форма запаздывающей части (16) проявляется в процессах, характеризующихся поведением затухающего гармонического осциллятора $[47,48]$.

\section{5. Кроссовер Иоффе-Регеля}

Знак подкоренного выражения в (14) определяет поведение комплексного модуля Юнга $E(\omega)$ и соотношение между упругостью и затуханием в системе. Частота

$$
\omega_{\mathrm{c}}=\frac{\varkappa}{2 a}
$$

разделяет колебания на две области: $\omega<\omega_{\mathrm{c}}$ и $\omega>\omega_{\mathrm{c}}$. Проанализируем характерные особенности колебаний в этих диапазонах частот.

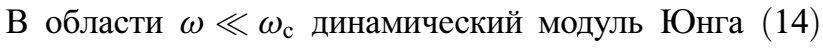
$E(\omega) \simeq \mathcal{x}$, и уравнение движения (15) описывает колебания упругих волн возмущения

$$
\ddot{u}_{\mathbf{q}}(t)+k \Delta u_{\mathbf{q}}(t)=0
$$

с коэффициентом жесткости $k=\Omega^{2} \varkappa$. Такие колебания являются фононами с линейным законом дисперсии $\omega(q)=q \sqrt{k}$.
Для нахождения закона дисперсии в области частот

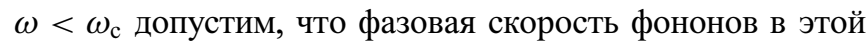
области частот определяется динамическим модулем Юнга (14): $v_{\mathrm{ph}}=\omega / q=\sqrt{\Omega^{2} E(\omega)}$. Из этого соотношения следует связь между частотой $\omega$ и волновым вектором $q$ :

$$
\omega(q)=\Omega^{2} a q \sqrt{2 q_{\mathrm{c}}^{2}-q^{2}}
$$

где волновой вектор $q_{\mathrm{c}}=\sqrt{\varkappa / 2 \Omega^{2} a^{2}}$ соответствует частоте $\omega_{\text {c. }}$ Такой закон дисперсии, полученный в более высоком приближении, хорошо описывает упругие колебания (фононы) во всем диапазоне частот $\omega<\omega_{\mathrm{c}}$.

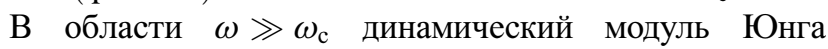
$E(\omega) \simeq i a \omega$, и уравнение движения (15) описывает диффузию профиля скоростей атомов

$$
\ddot{u}_{\mathbf{q}}(t)+D \Delta \dot{u}_{\mathbf{q}}(t)=0
$$

с коэффициентом диффузии $D=\Omega^{2} a$. При $\varkappa \ll 11$ такая диффузионная особенность колебаний сохраняется практически во всем диапазоне частот $\omega>\omega_{\mathrm{c}}$.

Таким образом, в области колебаний $\omega<\omega_{\mathrm{c}}$ распространяются хорошо определенные звуковые колебания, фононы, имеющие линейный закон дисперсии в низкочастотной области $\omega \ll \omega_{\text {c. }}$ В диапазоне частот

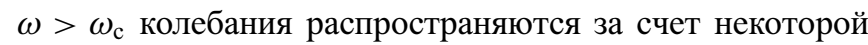
диффузионной передачи энергии от атома к атому в отсутствие явного переноса масс. Частота $\omega_{\mathrm{c}}$ разделяет эти две принципиально разные области колебаний. Это позволяет считать, что $\omega_{\mathrm{c}}$ соответствует частоте кроссовера Иоффе-Регеля $\omega_{\mathrm{IR}}$ при переходе от фононов с большой длиной свободного пробега к диффузионным колебаниям $[2,3]$.

\section{6. Плотность колебательных состояний}

Чтобы найти аналитическое выражение для плотности колебательных состояний $d$-мерной системы $g_{\text {рh }}(\omega)$ в фононной области частот $\omega<\omega_{\mathrm{c}}$, воспользуемся приближением $\omega / q=\sqrt{\Omega^{2} E(\omega)}$, в котором динамический модуль Юнга определяется соотношением (14):

$$
\begin{aligned}
g_{\mathrm{ph}}(\omega) & =\frac{s_{d}}{(2 \pi)^{d}} q^{d-1} \frac{\partial q}{\partial \omega} \\
& =\frac{s_{d} \omega^{d-1}}{(2 \pi \Omega \sqrt{a})^{d} \sqrt{\left(\omega_{\mathrm{c}}^{2}-\omega^{2}\right.}\left(\omega_{\mathrm{c}}+\sqrt{\omega_{\mathrm{c}}^{2}-\omega^{2}}\right)^{\frac{d}{2}-1}}
\end{aligned}
$$

где коэффициент $s_{d}=d(\sqrt{\pi})^{d} /[(d / 2) !]$ равен площади $d$-мерной гиперсферы единичного радиуса.

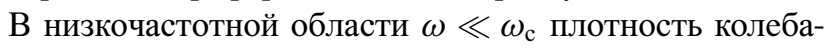
тельных состояний (21) имеет дебаевский вид

$$
g_{\mathrm{D}}(\omega)=\frac{s_{d} \omega^{d-1}}{(2 \pi \Omega \sqrt{\varkappa})^{d}} .
$$




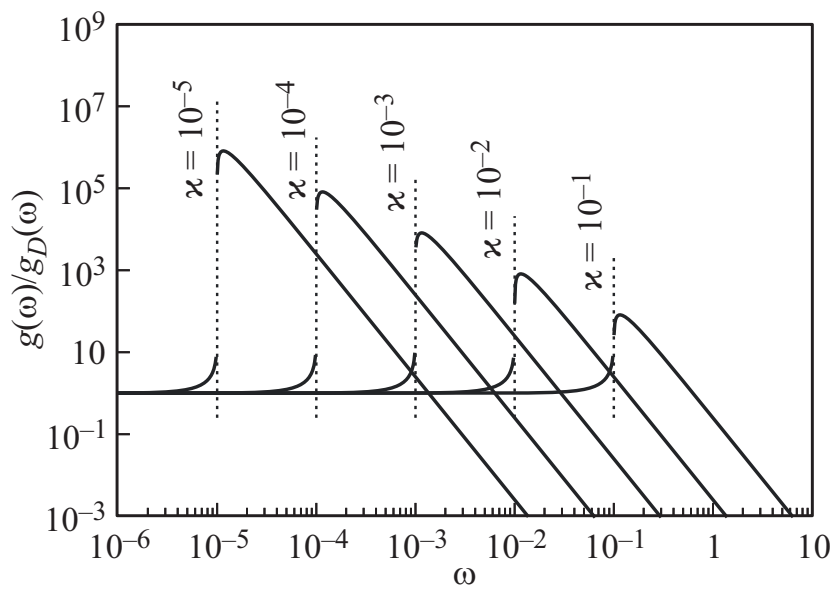

Приведенная плотность колебательных состояний области фононов $g_{\mathrm{ph}}(\omega) / g_{\mathrm{D}}(\omega)$ и области диффузионных колебаний $g_{\text {dif }}(\omega) / g_{\mathrm{D}}(\omega)$ для различных значений параметра $\varkappa$. Вертикальные пунктирные линии обозначают частоту кроссовера $\omega_{\mathrm{c}}$. Размерность системы $d=4$.

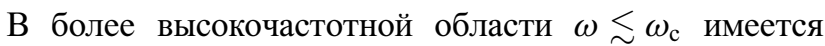
избыточный над дебаевским $g_{\mathrm{D}}(\omega) \propto \omega^{d-1}$ вклад в плотность состояний

$$
g_{\mathrm{ph}}(\omega)-g_{\mathrm{D}}(\omega) \sim \omega^{d+1} .
$$

Такой рост плотности колебательных состояний вблизи кроссовера Иоффе-Регеля $\omega_{\text {с }}$ является универсальным для систем с размерностью $d \geq 3$.

В диффузионной области колебаний $\omega>\omega_{\mathrm{c}}$ зависимость частоты от волнового вектора не является функциональной зависимостью, подобной дисперсионному соотношению для фононов (19). Тем не менее, из статистических свойств динамической матрицы и матрицы корреляций следует, что плотность колебательных состояний определяется мнимой частью динамического модуля Юнга [33]. С учетом выражения (14), плотность колебательных состояний в области диффузии колебаний принимает следующий вид:

$$
g_{\mathrm{dif}}(\omega)=\frac{2}{\pi \omega} \operatorname{Im} E(\omega)=\frac{2 a}{\pi \omega} \sqrt{\omega^{2}-\omega_{\mathrm{c}}^{2}} .
$$

Это уравнение соответствует закону МарченкоПастура [41] для классического некоррелированного ансамбля Вишарта. Правило сумм является необходимым требованием для существования плоско-волновых фононов, поэтому плотность колебательных состояний $g_{\text {dif }}$ безусловно описывает диффузионные колебания.

На рисунке показана нормированная на дебаевскую плотность колебательных состояний для случая $d=4$. Возникающий пик плотности состояний называется бозонным пиком. Как видно, с уменьшением параметра $\varkappa$, т.е. с ростом беспорядка в системе, высота бозонного пика увеличивается, и кроссовер между двумя областями колебаний становится более резким. Существует узкая область плавного перехода между $g_{\mathrm{ph}}(\omega)$ и $g_{\mathrm{dif}}(\omega)$, однако она мала по сравнению с $\omega_{\text {с }}$ при $\varkappa \ll 1$. При критических значениях $\omega=\omega_{\text {с }}$ выражения (21) и (24) имеют асимптотическую расходимость.

Плотность колебательных состояний определяет, в частности, затухание $\Gamma$, в следующем возможном виде [46]:

$$
\Gamma(\mathbf{q}, \omega)=\frac{\pi}{2} \omega_{\text {cor }}^{2}(\mathbf{q}) g(\omega) .
$$

В фононной области колебаний, несмотря на отсутствие мнимой части динамического модуля Юнга (14), затухание $\Gamma$ существует. $\mathrm{C}$ учетом выражения для плотности состояний (21) и дисперсионного соотношения (19), можно получить зависимость затухания колебаний от волнового вектора $q$ в фононной области $q<q_{\mathrm{c}}$ :

$$
\Gamma(q)=\frac{\pi}{2} \Omega^{2} q^{2} g_{\mathrm{ph}}=\frac{\pi s_{d}}{4 a(2 \pi)^{d}} q^{d+1} \frac{\sqrt{2 q_{\mathrm{c}}^{2}-q^{2}}}{q_{\mathrm{c}}^{2}-q^{2}} .
$$

Отсюда следует, что в широком диапазоне $q \ll q_{\text {c }}$ затухание определяется рэлеевским рассеянием фононов на беспорядке и дает вклад $\Gamma \propto q^{d+1}$. При критических значениях $q=q_{c}$ имеется асимптотическая расходимость.

В диффузионной высокочастотной области колебаний

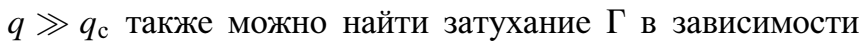
от волнового вектора $q$ :

$$
\Gamma(q)=\frac{\pi}{2} \Omega^{2} q^{2} g_{\text {dif }} \simeq D q^{2}
$$

где коэффициент диффузии $D=\Omega^{2} a$. Полученная зависимость $\Gamma \propto q^{2}$ для диффузионных колебаний является универсальной и не зависит от размерности системы. Эта диффузионная особенность согласуется с результатами экспериментов по неупругому рентгеновскому рассеянию в стеклах $[11,12]$ и с результатами метода молекулярной динамики для аморфного кремния [13].

\section{7. Заключение}

С помощью рассмотрения статистических свойств коррелированного ансамбля Вишарта случайных матриц, который описывает колебательные свойства аморфных твердых тел при учете их механической устойчивости и трансляционной инвариантности, в данной работе получены универсальные аналитические выражения, описывающие колебательные особенности таких систем вне зависимости от их размерности и локального расположения ближних атомов в них. Колебания неупорядоченных систем могут быть описаны с помощью уравнения движения усредненной проекции плоской волны $u_{\mathbf{q}}(t)$ с запаздывающей частью в виде динамического комплексного модуля Юнга $E(\omega)(11)$. При таком рассмотрении важным приближением является то, что низкочастотные колебания рассматриваемой среды обусловлены фононами с линейным законом дисперсии. 
В работе демонстрируется возникновение кроссовера Иоффе-Регеля в аморфных системах с размерностью $d \geq 3$ как кроссовера между фононами, описываемыми простым уравнением упругости для проекции смещений атомов (18), и диффузионными колебаниями, описываемыми уравнением диффузии для проекции скоростей $(20)$. Полученная частота кроссовера $\omega_{\mathrm{c}} \cong \omega_{\mathrm{IR}}(17)$ линейным образом коррелирует с параметром системы $\varkappa$, который характеризует макроскопическую жесткость неупорядоченной системы.

В случае малых значений $x \ll 1$ система обладает существенным беспорядком и демонстрирует избыточную плотность колебательных состояний $g(\omega)$ по сравнению с дебаевской плотностью состояний $g_{\mathrm{D}}(\omega)$. Это приводит к универсальному появлению бозонного пика в приведенной плотности колебательных состояний $g(\omega) / g_{\mathrm{D}}(\omega)$. Для иллюстрации полученных формул была рассмотрена система с размерностью $d=4$. Полученные колебательные свойства системы с такой размерностью являются важными для дальнейшего исследования, например, квазилокальных колебаний. В рамках подхода случайных матриц при рассмотрении негауссовой статистики распределения элементов матрицы $\hat{A}$ квазилокальные колебания могут быть получены и исследованы [43].

Затухание в области фононов демонстрирует их рэлеевское рассеяние на беспорядке и дает вклад $\Gamma \propto q^{d+1}$. Однако в более высокочастотной области выше кроссовера Иоффе-Регеля затухание $\Gamma \propto q^{2}$. Такая зависимость характерна для диффузионного гидродинамического демпфирования и имеет универсальный характер вне зависимости от размерности системы.

\section{Благодарности}

Автор благодарит Я.М. Бельтюкова за продуктивное обсуждение работы.

\section{Конфликт интересов}

Автор заявляет, что не имеет конфликта интересов.

\section{Список литературы}

[1] Д.А. Паршин. ФТТ 36, 7, 1809 (1994).

[2] P.B. Allen, J.L. Feldman. Phys. Rev. B 48, 17, 12581 (1993).

[3] P.B. Allen, J.L. Feldman, J. Fabian, F. Wooten. Phil. Mag. B 79, 11, 1715 (1999).

[4] B. Rufflé, G. Guimbretiére, E. Courtens, R. Vacher, G. Monaco. Phys. Rev. Lett. 96, 4, 045502 (2006).

[5] B. Rufflé, D.A. Parshin, E. Courtens, R. Vacher. Phys. Rev. Lett. 100, 1, 015501 (2008).

[6] V.L. Gurevich, D.A. Parshin, H.R. Schober. Phys. Rev. B 67, 9, 094203 (2003).

[7] V.K. Malinovsky, A.P. Sokolov. Solid State Commun. 57, 9, 757 (1986).

[8] A. Wischnewski, U. Buchenau, A.J. Dianoux, W.A. Kamitakahara, J.L. Zarestky. Phil. Mag. B 77, 2, 579 (1998).
[9] P. Benassi, M. Krisch, C. Masciovecchio, V. Mazzacurati, G. Monaco, G. Ruocco, F. Sette, R. Verbeni. Phys. Rev. Lett. 77, 18, 3835 (1996).

[10] T. Ohsaka, T. Ihara. Phys. Rev. B 50, 13, 9569 (1994).

[11] F. Sette, M.H. Krisch, C. Masciovecchio, G. Ruocco, G. Monaco. Sci. 280, 5369, 1550 (1998).

[12] G. Ruocco, F. Sette. J. Phys.: Condens. Matter 13, 41, 9141 (2001).

[13] J.K. Christie, S.N. Taraskin, S.R. Elliott. J. Non-Cryst. Solids 353, 22-23, 2272 (2007).

[14] V. Vitelli, N. Xu, M. Wyart, A.J. Liu, S.R. Nagel. Phys. Rev. E 81, 2, 021301 (2010).

[15] S. Kojima, Y. Matsuda, M. Kodama, H. Kawaji, T. Atake. Chin. J. Phys. 49, 1, 414 (2011).

[16] W. Steurer, A. Apfolter, M. Koch, W.E. Ernst, E. Søndergård, J.R. Manson, B. Holst. Phys. Rev. B 78, 4, 045427 (2008).

[17] W. Steurer, A. Apfolter, M. Koch, W.E. Ernst, B. Holst, E. Søndergård, J.R. Manson. Phys. Rev. Lett. 99, 3, 035503 (2007).

[18] L. Zhang, J. Zheng, Y. Wang, L. Zhang, Z. Jin, L. Hong, Y. Wang, J. Zhang. Nature Commun. 8, 1, 67 (2017).

[19] L. Wang, A. Ninarello, P. Guan, L. Berthier, G. Szamel, E. Flenner. Nature Commun. 10, 1, 26 (2019).

[20] M. Wyart. EPL (Europhys. Lett.) 89, 6, 64001 (2010).

[21] H. Mizuno, H. Shiba, A. Ikeda. PNAS 114, 46, E9767 (2017).

[22] A.I. Chumakov, G. Monaco, A. Monaco, W.A. Crichton, A. Bosak, R. Rüffer, A. Meyer, F. Kargl, L. Comez, D. Fioretto, H. Giefers, S. Roitsch, G. Wortmann, M.H. Manghnani, A. Hushur, Q. Williams, J. Balogh, K. Parliński, P. Jochym, P. Piekarz. Phys. Rev. Lett. 106, 22, 225501 (2011).

[23] S.N. Taraskin, Y.L. Loh, G. Natarajan, S.R. Elliott. Phys. Rev. Lett. 86, 7, 1255 (2001).

[24] D.A. Parshin, H.R. Schober, V.L. Gurevich. Phys. Rev. B 76, 6, 064206 (2007).

[25] W. Götze, M.R. Mayr. Phys. Rev. E 61, 1, 587 (2000).

[26] M. Baggioli, R. Milkus, A. Zaccone. Phys. Rev. E 100, 6, 062131 (2019).

[27] T. Grigera, V. Martin-Mayor, G. Parisi, P. Verrocchio. J. Phys.: Condens. Mater 14, 9, 2167 (2002).

[28] M.L. Manning, A.J. Liu. EPL 109, 3, 36002 (2015).

[29] Y.M. Beltukov, V.I. Kozub, D.A. Parshin. Phys. Rev. B 87, 13, 134203 (2013).

[30] Y.M. Beltukov, D.A. Parshin. Phys. Solid State 53, 1, 151 (2011).

[31] Y.M. Beltukov, D.A. Parshin. JETP Lett. 104, 8, 552 (2016).

[32] D.A. Conyuh, Y.M. Beltukov. Phys. Solid State 62, 4, 689 (2020).

[33] D.A. Conyuh, Y.M. Beltukov. Phys. Rev. E 103, 4-1, 042608 (2021).

[34] U. Buchenau, Y.M. Galperin, V. Gurevich, D. Parshin, M. Ramos, H. Schober. Phys. Rev. B 46, 5, 2798 (1992).

[35] M. Shimada, H. Mizuno, A. Ikeda. Soft Matter 16, 31, 7279 (2020).

[36] A.A. Maradudin, E.W. Montroll, G.H. Weiss, I. Ipatova. Theory of lattice dynamics in the harmonic approximation, vol. 3. Academic Press, N. Y. (1963).

[37] Y.M. Beltukov. JETP Lett. 101, 5, 345 (2015).

[38] C.S. O’Hern, L.E. Silbert, A.J. Liu, S.R. Nagel. Phys. Rev. E 68, 1, 011306 (2003).

[39] J.C. Maxwell. Phil. Mag. J. Sci. 27, 182, 294 (1864).

[40] J. Wishart. Biometrika 20A, 1/2, 32 (1928). 
[41] В.А. Марченко, Л.А. Пастур. Матем. сборник 72, 4, 507 (1967).

[42] J. Bun, J.P. Bouchaud, M. Potters. Phys. Reports 666, 1 (2017).

[43] D.A. Conyuh, Y.M. Beltukov. Phys. Rev. B 103, 10, 104204 (2021).

[44] Z. Burda, A. Görlich, J. Jurkiewicz, B. Wacław. Eur. Phys. J. B 49, 3, 319 (2006).

[45] I.J. Zucker. J. Status Phys. 145, 3, 591 (2011).

[46] D.A. Conyuh, Y.M. Beltukov. JETP Lett. 112, 8, 513 (2020).

[47] A.V. Mokshin, R.M. Yulmetyev, P. Hänggi. Phys. Rev. Lett. 95, 20, 200601 (2005).

[48] A.V. Mokshin. arXiv:1312.2085 [cond-mat, physics:physics] (2013).

Редактор Е.В. Толстякова 